denzbasierte, leitliniengerechte Therapie für KHK-Patienten nach einer PCI.“ Die Leitlinien empfehlen moderates Ausdauertraining mindestens drei bis fünfmal die Woche für 30 bis 60 Minuten, unterhalb der Angina-Schwelle.

So überzeugend der Nutzen von körperlichem Training auch bei Herzinsuffizienz und nach PCI erbracht wurde, die Langzeitdaten weisen auf ein Kernproblem: schlechte Compliance. Die Folgen: Alte Verhaltensweisen kehren wieder und mit ihnen die Risikofaktoren. Deshalb wird gefordert: „Körperliches Training sollte Teil eines Langzeitpräventionsprogramm sein, das verschiedene Risikofaktoren kontrolliert."

Wie aber sollen die Patienten bei der Stange gehalten werden? Hambrecht plädiert für neue Konzepte. Langzeitpräventionsprogramme koordiniert durch Präventionsassistent(innen). Dass dieses Konzept funktionieren kann, zeigte die IPP-Studie (Intensives Präventions-Programm). Die 12-Monats-Daten sind unmissverständlich, das IPP ist der Standardversorgung in allen geprüften Bereichen überlegen.

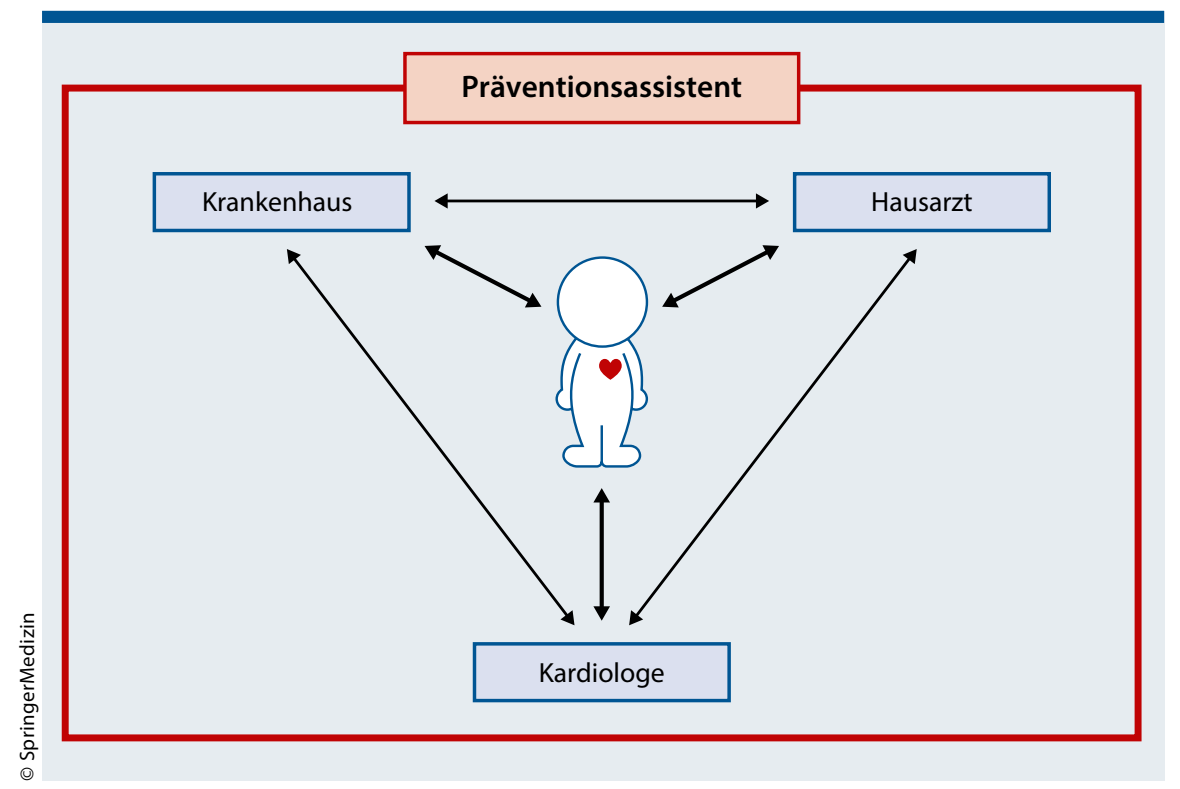

Abb. 1: Der Präventionsassistent sollte bei Patienten nach einer Stentimplantation als Schnittstelle zwischen Kardiologe, Hausarzt und Klinik fungieren.

Das neue Konzept integriert den Präventionsassistenten in ein Netzwerk zwischen Krankenhaus, Hausarzt und Kardiologen, die sich gemeinsam um ein
Präventionsprogramm für den individuellen PCI-Patienten kümmern (Abb. 1). Zur Nachahmung empfohlen.

Dr. med. Jochen Aumiller

\title{
Neue PAVK-Leitlinie mit Überraschungen
}

\section{Seit September 2017 gibt es eine neue europäische Leitlinie zur Behandlung der peripheren Verschlusserkrankung (PAVK). Sie hat essenzielle Bedeutung für rund 4,5 Millionen Deutsche, die unter einer PAVK leiden.}

$P_{b}^{A}$ AVK-Patienten, das ist seit langem bekannt, sind auch kardiale Hochrisikopatienten. Jeder Dritte hat eine KHK, ebenso viele weisen Karotisplaques auf. Dies bedeutet laut Prof. Christiane Tiefenbacher von der Westfälischen Wilhelms-Universität in Münster, dass es bei der PAVK nicht nur um die Beine, sondern auch um Herz und Hirn gehe.

Die neue Leitlinie der europäischen Gesellschaft für Kardiologie (ESC) überträgt das Konzept des Herzteams auf die Gefäße. Ein Gefäßteam aus Kardiologen, Angiologen und Gefäßchirurgen sollte entscheiden, welche Behandlungsmethode für den individuellen Patienten am besten geeignet ist. Neben dieser systemischen Anregung gehen die Leitlinien auf ganz konkrete Punkte ein:

_Lipidsenkende Therapie mit Statinen, sofern sie vertragen werden. Dadurch kann auch die Gehstrecke verbessert werden.

_Thrombozytenhemmer bzw. Antikoagulation: Neu für Europa ist die Degradierung von ASS zugunsten von Clopidogrel.

- PAVK-Patienten, die unter Marcumar oder neuen oralen Antikoagulanzien stehen, sollten wegen des erhöhten Blutungsrisikos Thrombozytenhemmer weglassen.

- Bei asymptomatischen Karotisstenosen steht nicht mehr der chirurgische Eingriff, sondern die für den Patienten schonendere katheterge- stützte Stent-implantation im Vordergrund.

Bei der Therapie der Beinarterien sind die Katheterinterventionen auf dem Vormarsch. Operative Verfahren sollen vor allem in komplexen Situationen, etwa bei starker Gefäßverkalkung, zum Zuge kommen. In der Leitlinie erkennt man den mahnenden Zeigefinger bzgl. der Kathetereingriffe: Sie sollten nur in Zentren durchgeführt werden, die über „sehr erfahrene Fachleute“ verfügen.

Ist der Zustand der Venen im Unterschenkel gut, kann nach Ansicht von Tiefenbacher auch eine Bypass-Operation durchgeführt werden, bei der Venen als Überbrückung der verschlossenen Arterien dienen.

Und noch eine allgemeine Ermahnung: Bei PAVK-Patienten soll der Angiologe systematisch darauf zu achten, ob es Indizien für eine KHK oder Herzinsuffizienz gibt. Umgekehrt wird den Kardiologen nahegelegt, ihre Patienten darauf zu untersuchen, ob eine PAVK vorliegt. Dr. med. Jochen Aumiller 